\title{
A diabetic lady with difficulty in breathing
}

\author{
Seng Wee Cheo, Khai Lip Ng, Qin Jian Low \\ Cheo SW, Ng KL, Low QJ. A diabetic lady with difficulty in breathing. Malays Fam Physician. 2021;16(3);126-128. https://doi.org/10.51866/tyk1188
}

\section{Keywords:}

Tuberculosis, air crescent

sign, dyspnea

\section{Authors:}

\section{Cheo Seng Wee \\ (Corresponding author) \\ MRCP (UK) \\ Deparment of Internal Medicine Hospital Lahad Datu, Peti Bersurat Lahad Datu, Sabah, Malaysia. Email: cheosengwee@gmail.com}

\section{Ng Khai Lip}

MRCP (UK)

Department of Respiratory Medicine Hospital Queen Elizabeth, 13a, Jalan Penampang, Kota Kinabalu, Sabah Malaysia.

\section{Low Qin Jian}

MRCP (UK)

Department of Internal Medicine Hospital Sultanah Nora Ismail, Jalan Korma, Taman Soga, Batu Pahat Johor, Malaysia

\section{Introduction}

Dyspnoea is a common respiratory symptom that clinicians encounter in daily clinical practice. It is defined as a subjective experience of breathing discomfort that consists of qualitatively distinct sensations that vary in intensity. ${ }^{1}$ The various causes of dyspnoea can be broadly divided into cardiogenic causes, respiratory causes, neuromuscular causes and others. ${ }^{2}$ In approaching a patient who complains of dyspnoea, we must systemically evaluate him/her to obtain a definitive diagnosis. Investigations that can be performed include arterial blood gas analysis, electrocardiography, echocardiography and chest radiograph. Here, we illustrate a patient who complained of breathing difficulty.

\section{Case Presentation}

A 46-year-old Filipino woman with noninsulin-dependent diabetes mellitus and previous pulmonary tuberculosis (4 years ago prior) presented to a local health clinic with chronic productive cough with greenish sputum and fever (3 months in duration) associated with loss of appetite and weight loss. She denied shortness of breath or haemoptysis. On examination, there was bronchial breathing in the left upper lobe. Other systemic examinations were unremarkable. Her sputum was positive for acid-fast bacilli and she was started on antituberculous therapy.

Two months later, she presented to the clinic with worsening dyspnoea and fever (1 week in duration). She denied haemoptysis. On examination, she was alert and mildly tachypneic. Her respiratory examinations showed bronchial breathing in the left upper zone and dullness on percussion. Other systemic examinations were unremarkable. Her full blood count showed a haemoglobin of $11.9 \mathrm{~g} / \mathrm{dl}$, total white blood cell count of $16.2 \times 109 / \mathrm{L}$ and platelet count of $210 \times 109 / \mathrm{L}$. Her renal profile and liver function test were normal. Arterial blood gas showed no evidence of respiratory failure. A chest radiograph was also performed (Figure 1).

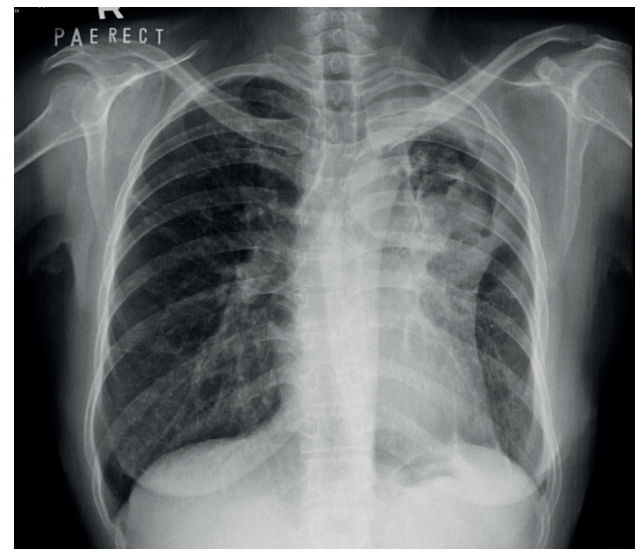

Figure 1. Chest radiograph of the patient

\section{Questions and Answers:}

1. Describe the chest radiograph.

Figure 1 presents the PA erect view chest radiograph of the patient, which shows a radiopaque cavitary lesion at the left upper lobe surrounded by a crescentshaped radiolucency typical of an air crescent sign. The right lung field, ribs and heart appear normal.

2. What is the most likely diagnosis and differential diagnosis?

The most likely diagnosis is pulmonary aspergilloma. The fundamental diagnosis is based on the history of previous tuberculosis and the current chest radiograph showing an air crescent sign. The diagnosis is confirmed by Monod's sign (see below). Differential diagnoses to consider include pulmonary tuberculosis, Rasmussen aneurysm, lung abscess, hydatid cyst and cavitary lung cancer. ${ }^{3}$

3. What other investigations can be performed?

a) Sputum acid-fast bacilli - To check for active pulmonary tuberculosis.

b) Sputum for culture and sensitivity - To culture for bacterial, mycobacterial and fungal organisms.

c) Serum Aspergillus IgG - To confirm the diagnosis of aspergillosis. ${ }^{4}$ 
d) Bronchoscopy - In patients with a negative fungal culture, bronchoscopy should be performed to collect a bronchial washing sample and exclude malignancy. ${ }^{5}$

e) Computed tomography of the thorax - To further characterise the air crescent sign and differentiate between the differential diagnoses. Contrastenhanced CT of the thorax may help to identify Rasmussen aneurysm and may also help in identifying cavitary lung cancer. ${ }^{6}$

\section{Outline the management plan}

Most aspergillomas are asymptomatic and their cavity is often isolated. Thus, there is a limited role for systemic antifungals. Patients should be followed up and monitored for disease complications (e.g., infections and haemoptysis). A single symptomatic lesion is best treated with surgical resection. ${ }^{4}$ Patients who develop massive haemoptysis may require embolisation or surgery.

\section{Case Progress}

Returning to our case, the patient's chest radiograph showed a radiopaque cavitary lesion at the left upper lobe, which was surrounded by a crescentshaped radiolucency suggestive of an air crescent sign. The patient subsequently had a chest radiograph performed in the decubitus position, in which the cavitary lesion showed a change in position. This is typical of Monod's sign, thus confirming the diagnosis of aspergilloma (Figure 2). Her sputum cultures were negative for bacterial, mycobacterial and fungal organisms.

She was then counselled for serum Aspergillus IgG, bronchoscopy and computed tomography of the thorax. She refused further investigation due to financial reasons. She was treated with a course of antibiotics for superimposed bacterial infection and then improved. On follow-up after completion of antituberculous therapy, her chest radiograph remained the same and she did not have any haemoptysis.

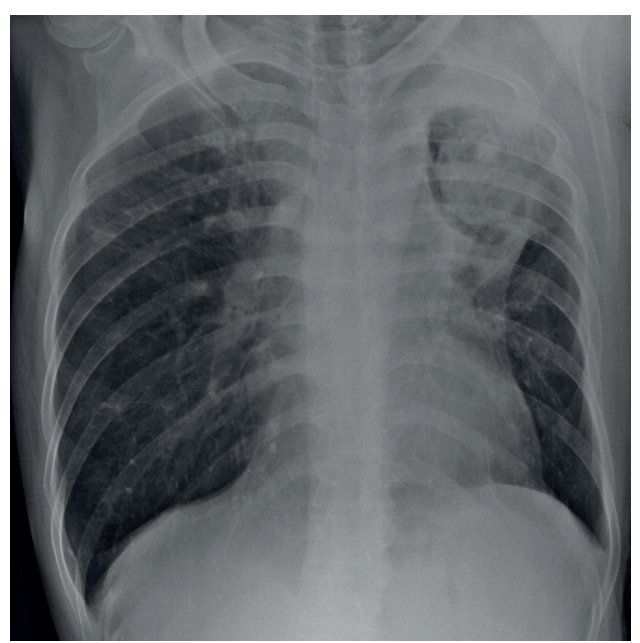

Figure 2. Chest radiograph showing Monod's sign.

\section{Discussion}

An air crescent sign is a radiological sign involving a crescent-shaped radiolucency surrounding a parenchymal consolidation or nodular opacity. ${ }^{7}$ Monod's sign describes the mass or consolidation move to the dependent area. ${ }^{8}$ An air crescent sign can be seen in the radiograph or computed tomography. By far, the most common cause of an air crescent sign is aspergilloma. Differential diagnoses could include pulmonary tuberculosis, pulmonary hydatid cyst, Rasmussen aneurysm and lung carcinoma. ${ }^{?}$

In light of previous tuberculosis and the presence of an air crescent sign, we believe that our patient most likely had aspergilloma. Moreover, the air crescent sign persisted before and after treatment with antibiotics and antituberculous therapy. On the other hand, Aspergillus fumigatus is the most common Aspergillus species that causes disease in humans. Pulmonary aspergillosis usually occurs in patients with underlying lung disease (e.g., sarcoidosis, cystic fibrosis and old tuberculosis). In old tuberculosis, there are residual cavities from a previous infection that aspergillomas tend to form within. ${ }^{10}$ In a British study, it was estimated that chronic pulmonary aspergillosis complicates $4.9-6.3 \%$ of all previously treated tuberculosis. Notably, it is more common in patients with cavitary lung lesions. ${ }^{11}$

Aspergilloma usually occurs in immunocompetent patients, with most aspergilloma being asymptomatic. Symptoms are non-specific, with some patients presenting 
with cough, fever, chest pain and hemoptysis. ${ }^{4}$ Aspergilloma can typically be managed with expectant management. However, one must remember that massive haemoptysis is a potentially life-threatening complication and the necessary steps must be taken to treat it. Systemic anti-fungals have a limited role since the cavity is often isolated.

In conclusion, although an air crescent sign is an important clinical sign, it is not pathognomonic for aspergilloma. Thus, we must always interpret this radiological sign according to the clinical presentation of patients.

\section{Conflicts of Interest}

The author declared that they have no conflicts of interest.

\section{Funding}

The author received no financial support for this publication.

\section{How does this paper make a difference to general practice?}

The chest radiograph is widely used in clinical practice. As medical practitioners, we must be able to recognise important radiological signs and correlate them with the clinical presentation of patients. Through this case, we aim to illustrate the importance of the air crescent sign. The differential diagnoses of air crescent sign include pulmonary aspergilloma, cavitary lung cancer, Rasmussen aneurysm, hydatid cyst and lung abscess.

\section{References}

1. Parshall MB, Schwartzstein RM, Adams L, et al. An official American Thoracic Society statement: update on the mechanisms, assessment, and management of dyspnea. Am J Respir Crit Care Med. 2012;185(4):435-452. doi:10.1164/rccm.201111-2042ST

2. Berliner D, Schneider N, Welte T, Bauersachs J. The Differential Diagnosis of Dyspnea. Dtsch Arztebl Int. 2016;113(49):834-845. doi:10.3238/arztebl.2016.0834

3. Tseng YY, Chen $\mathrm{CH}$. Air crescent sign: not always due to fungal infection. QJM. 2015;108(3):255-256. doi:10.1093/qjmed/ hcu 185

4. Ofori A, Steinmetz AR, Akaasi J, et al. Pulmonary aspergilloma: An evasive disease. Int J Mycobacteriol. 2016;5(2):235-239. doi:10.1016/j.ijmyco.2016.03.002
5. Zmeili OS, Soubani AO. Pulmonary aspergillosis: a clinical update. QJM. 2007;100(6):317-334. doi:10.1093/qjmed/ hcm 035

6. Sevilha JB, Rodrigues RS, Barreto MM, Zanetti G, Hochhegger B, Marchiori E. Infectious and Non-Infectious Diseases Causing the Air Crescent Sign: A State-ofthe-Art Review. Lung. 2018;196(1):1-10. doi:10.1007/s00408-017-0069-3

7. Abramson S. The air crescent sign. Radiology. 2001;218(1):230-232. doi:10.1148/ radiology.218.1.r01ja19230

8. Sharma S, Dubey SK, Kumar N, Sundriyal D. 'Monod' and 'air crescent' sign in aspergilloma. BMJ Case Rep. 2013;2013:bcr2013200936. Published 2013 Sep 13. doi:10.1136/bcr-2013-200936
9. Fred HL, Gardiner CL. The air crescent sign: causes and characteristics. Tex Heart Inst J. 2009;36(3):264-265.

10. Sugino K, Hasegawa C, Sano G, Shibuya K, Homma S. Pathophysiological study of chronic necrotizing pulmonary aspergillosis. Jpn J Infect Dis. 2008;61(6):450-453.

11. Page ID, Byanyima R, Hosmane $S$, et al. Chronic pulmonary aspergillosis commonly complicates treated pulmonary tuberculosis with residual cavitation. Eur Respir J. 2019;53(3):1801184. Published 2019 Mar 18. doi:10.1183/13993003.01184-2018 